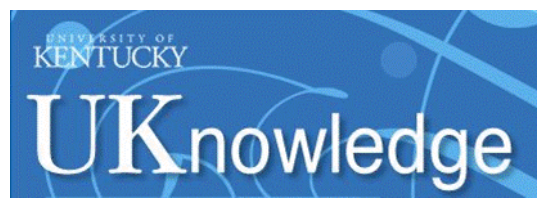

University of Kentucky

UKnowledge

8-19-2016

\title{
Interaction-Induced Dirac Fermions from Quadratic Band Touching in Bilayer Graphene
}

\author{
Sumiran Pujari \\ University of Kentucky, sumiran.pujari@uky.edu \\ Thomas C. Lang \\ University of Innsbruck, Austria \\ Ganpathy Murthy \\ University of Kentucky, ganpathy.murthy@uky.edu \\ Ribhu K. Kaul \\ University of Kentucky, ribhu.kaul@uky.edu
}

Follow this and additional works at: https://uknowledge.uky.edu/physastron_facpub

Part of the Condensed Matter Physics Commons

Right click to open a feedback form in a new tab to let us know how this document benefits you.

\section{Repository Citation}

Pujari, Sumiran; Lang, Thomas C.; Murthy, Ganpathy; and Kaul, Ribhu K., "Interaction-Induced Dirac Fermions from Quadratic Band Touching in Bilayer Graphene" (2016). Physics and Astronomy Faculty Publications. 441.

https://uknowledge.uky.edu/physastron_facpub/441

This Article is brought to you for free and open access by the Physics and Astronomy at UKnowledge. It has been accepted for inclusion in Physics and Astronomy Faculty Publications by an authorized administrator of UKnowledge. For more information, please contact UKnowledge@lsv.uky.edu. 


\title{
Interaction-Induced Dirac Fermions from Quadratic Band Touching in Bilayer
} Graphene

\author{
Digital Object Identifier (DOI) \\ https://doi.org/10.1103/PhysRevLett.117.086404 \\ Notes/Citation Information \\ Published in Physical Review Letters, v. 117, issue 8, 086404, p. 1-5. \\ (C) 2016 American Physical Society
}

The copyright holder has granted permission for posting the article here. 


\title{
Interaction-Induced Dirac Fermions from Quadratic Band Touching in Bilayer Graphene
}

\author{
Sumiran Pujari, ${ }^{1}$ Thomas C. Lang, ${ }^{2}$ Ganpathy Murthy, ${ }^{1}$ and Ribhu K. Kaul ${ }^{1}$ \\ ${ }^{1}$ Department of Physics and Astronomy, University of Kentucky, Lexington, Kentucky 40506-0055, USA \\ ${ }^{2}$ Institute for Theoretical Physics, University of Innsbruck, 6020 Innsbruck, Austria
}

(Received 26 April 2016; published 19 August 2016)

\begin{abstract}
We revisit the effect of local interactions on the quadratic band touching (QBT) of the Bernal honeycomb bilayer model using renormalization group (RG) arguments and quantum Monte Carlo (QMC) simulations. We present a RG argument which predicts, contrary to previous studies, that weak interactions do not flow to strong coupling even if the free dispersion has a QBT. Instead, they generate a linear term in the dispersion, which causes the interactions to flow back to weak coupling. Consistent with this RG scenario, in unbiased QMC simulations of the Hubbard model we find compelling evidence that antiferromagnetism turns on at a finite $U / t$ despite the $U=0$ hopping problem having a QBT. The onset of antiferromagnetism takes place at a continuous transition which is consistent with $(2+1) \mathrm{D}$ Gross-Neveu criticality. We conclude that generically in models of bilayer graphene, even if the free dispersion has a QBT, small local interactions generate a Dirac phase with no symmetry breaking and that there is a finite-coupling transition out of this phase to a symmetry-broken state.
\end{abstract}

DOI: 10.1103/PhysRevLett.117.086404

The interplay of band topology and interactions has taken center stage in condensed matter physics. The interest in band topology was rekindled by the discovery of graphene, where two bands touch at linearly dispersing Dirac points [1]. Short-range interactions are known to be irrelevant at the Dirac fixed point, making the linear band touching a stable many-body phenomenon for weak interactions. Strong interactions trigger an instability to a broken-symmetry state [2-4].

Subsequently, it was pointed out that bilayer graphene harbors a quadratic band touching (QBT) at half filling in the nearest-neighbor hopping model [5]. Renormalization group studies have shown that interactions are marginally relevant at a QBT [6]. This led to predictions of the stabilization of a symmetry-broken state even for arbitrarily weak short-range interactions in bilayer graphene $[7,8]$ in contrast to single-layer graphene. The nature of the symmetry-broken state depends on the form of the interactions, and a plethora of phases have been proposed (see, e.g., [7-11]). The Bernal honeycomb bilayer (BHB) lattice with Hubbard interactions was studied as an example of this kind of ordering [12]. Quantum Monte Carlo simulation studies concluded that this model was Néel ordered for all values of the coupling $U / t$ consistent with the predictions of weak coupling [8] and the functional renormalization group (RG) [12].

On the inclusion of certain symmetry-preserving hoppings beyond the shortest range on the BHB (called trigonal warping), a linear term in the dispersion is present at low energies, resulting in Dirac nodes [5]. It has been generally assumed that if the trigonal warping terms are very weak microscopically, for practical purposes they can be neglected-they cause the symmetry-breaking instability to appear only at a small interaction strength controlled by their size [13], leading to the RG flow in Fig. 1(a). Here we present arguments and evidence that neglecting linear terms in the dispersion is not justified in determining the fate of short-range interactions at the quadratic band touching in bilayer graphene. Even in a model BHB system, where the trigonal warping terms are microscopically zero and there is a true QBT in the free problem, interactions generate the linear term in a RG flow. At weak coupling, we thus conclude that interactions cause the emergence of a Dirac phase from a QBT in bilayer graphene. The instability to a symmetry-broken ordered state then takes place at a finite
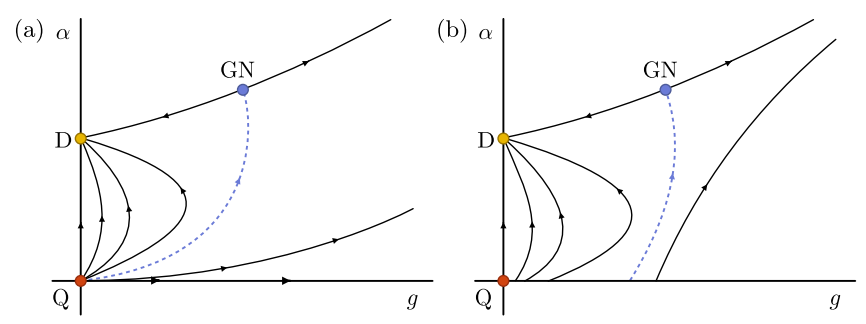

FIG. 1. Two schematics for the renormalization group flows for the Bernal stacked bilayer in the space of $\alpha$ (linear term in the dispersion) and $g$ (generic quartic interactions) [cf. Eq. (1) for definitions]. The fixed points are $Q$, quadratic band touching; $D$, Dirac linear dispersion; and GN, Gross-Neveu. (a) The RG flow implied by previous studies. In this scenario, weak interactions always flow to strong coupling when one begins with a QBT, i.e., along $\alpha=0$. (b) RG flow advocated for in this work, when $\alpha$ arises in a RG flow due to interactions. Note that crucially in (b), $\alpha$ is generated even when one begins on the $\alpha=0$ line. The point marked as $D$ indicates one of a set of fixed points with $\alpha \neq 0$ which all have a linear Dirac dispersion at low energy but different details of the electronic structure. 
strength of electron-electron interactions [cf. Fig. 1(b)]. Field theoretic arguments suggest that this transition, if continuous, would be of the Gross-Neveu (GN) type [2]. As a concrete test of our RG scenario, we present QMC simulations of the Hubbard interaction on the bilayer graphene lattice. We show that, despite the model having a QBT at $U=0$, interactions cause Néel ordering only at a finite value of $U_{c} \approx 2.6$. The phase transition between the non-Néel and Néel phases is fully consistent with the GN universality class, consistent with Fig. 1(b).

$R G$ argument.-In the absence of spin-orbit coupling, symmetries guarantee that two spin-degenerate low-energy bands touch at the $\mathbf{K}$ and $\mathbf{K}^{\prime}$ points. Expanding around the $\mathbf{K}$ point, a symmetry-based study [5,8] of allowed dispersions for the part quadratic in the fermionic operators $\Psi$ of the Hamiltonian density has the following form up to second order in $\mathbf{k}$ :

$$
\begin{aligned}
h^{(2)}(\mathbf{K}+\mathbf{k}) & =\Psi_{\mathbf{K}+\mathbf{k}}^{\dagger}\left\{\operatorname{Re}[\phi(\mathbf{k})] \sigma^{x}+\operatorname{Im}[\phi(\mathbf{k})] \sigma^{y}\right\} \Psi_{\mathbf{K}+\mathbf{k}}, \\
\phi(\mathbf{k}) & \equiv \alpha\left(k_{x}+i k_{y}\right)+\beta\left(k_{x}-i k_{y}\right)^{2}+\mathcal{O}\left(k^{3}\right) .
\end{aligned}
$$

The crucial point for our study is that generically a linear term $\alpha$ is allowed by symmetries. At quartic order, $H^{(4)}$ has nine independent symmetry-allowed terms of the form $\Psi^{\dagger} \Psi^{\dagger} \Psi \Psi$ with coupling constants $g_{i}(i \in[1,9])$ [8].

From the RG point of view, the quadratic band touching is a fixed point, called $Q\left(\alpha, g_{i}=0\right)$, which has $z=2$. Power counting shows $\alpha$ is relevant at $Q$ and the $g_{i}$ are marginal-all other couplings are irrelevant [14]. A oneloop calculation finds that $g_{i}$ are generically marginally relevant [7,8]; $Q$ is hence a multicritical point in the $\mathrm{RG}$ sense. We now ask how $Q$ is affected when we add only $g_{i}$ but no $\alpha$ (this corresponds to adding interactions to a free dispersion with a true QBT). Wilsonian RG tells us that all terms that are allowed by symmetries will appear as we integrate high-energy degrees of freedom. Since no symmetry forces $\alpha=0$ in the dispersion for the Bernal stacked bilayer, it must be generated. Since at $Q$ the linear term is relevant and the interactions are only marginal, at weak coupling the linear term will always grow faster, and hence the RG flows will take us to a fixed point with Dirac fermions (see [15] for more details). At this fixed point called $D$, interactions are irrelevant, and hence it is a stable phase of matter (just like in single-layer graphene). Once the $g_{i}$ cross a finite order-one strength, they will eventually win over the generated $\alpha$ and a flow to strong coupling will ensue. The separatrix between the flow to weak and strong coupling can flow to an intermediate coupling fixed point, the GN universality class-Fig. 1(b). We note that, in models with higher symmetry than the BHB, the QBT can be symmetry protected $[6,16]$, and then weak interactions will flow to strong coupling-Fig. 1(a) is the correct flow.

Hubbard model.-Although our RG arguments apply independently of the form of short-range interactions [14], to provide a concrete lattice realization of our predicted emergence of the Dirac phase from the QBT we present a detailed numerical study of this phenomena in the Hubbard model on the Bernal stacked honeycomb bilayer. Defining the electron creation operator on sublattice $A(B)$, site $i$, spin $\sigma$, and layer $\ell$ as $a_{i \sigma \ell}^{\dagger}\left(b_{i \sigma \ell}^{\dagger}\right)$, the Hamiltonian takes the form [17]

$$
\begin{aligned}
H_{\mathrm{BSB}}= & -t \sum_{\langle i j\rangle \ell} a_{i \sigma \ell}^{\dagger} b_{j \sigma \ell}-t_{\perp} \sum_{i} a_{i \sigma 2}^{\dagger} b_{i \sigma 1}+\text { H.c. } \\
& +U \sum_{i, \ell}\left(a_{i \uparrow \ell}^{\dagger} a_{i \uparrow \ell} a_{i \downarrow \ell}^{\dagger} a_{i \downarrow \ell}+b_{i \uparrow \ell}^{\dagger} b_{i \uparrow \ell} b_{i \downarrow \ell}^{\dagger} b_{i \downarrow \ell}\right),
\end{aligned}
$$

where $\langle i j\rangle$ are bipartite nearest neighbors on the honeycomb layers. We choose $t=t_{\perp}=1$, fixed throughout the Letter (results for $t / t_{\perp} \neq 1$ are presented in Ref. [15]). This model has been studied previously, where it was found that for $U / t>3$ the model has Néel order. For $U / t<3$, although no numerical evidence was found for the presence of Néel order in extrapolations of the order parameter, it was assumed based on RG calculations that the model was weakly Néel ordered [12], supporting the RG flow in Fig. 1(a) on the $\alpha=0$ axis. We have already presented arguments that invalidate this conclusion and suggest that the correct RG flow for the Bernal stacked bilayer (BSB) is Fig. 1(b). To further back up our RG argument, we now present clear numerical evidence that, contrary to the conclusion of Ref. [12], $H_{\mathrm{BSB}}$ has a finite coupling phase transition at which magnetism appears [18].

To connect $H_{\mathrm{BSB}}$ with the long-wavelength description [Eq. (1)] with only $t, t_{\perp}$ hopping and $U=0$, the system realizes Eq. (1) with $\alpha=0$, and a QBT arises described by the fixed point $Q$. Crucially, as noted earlier, there is no symmetry that protects $\alpha=0$. Indeed, trigonal hopping of the form $-t_{3} \sum_{\langle i j\rangle \sigma} b_{i \sigma 2}^{\dagger} a_{j \sigma 1}$ preserves the symmetries of the bilayer and nonetheless has an $\alpha$ term, which makes the dispersion at low energies linear [5].

We emphasize that all our simulations are carried out with $H_{\mathrm{BSB}}$ itself and with no $t_{3}$ hopping. Thus, $H_{\mathrm{BSB}}$ can be thought of as lying along the $\alpha=0$ axis in Figs. 1(a) and 1(b). The two RG scenarios (a) and (b) make strikingly different predictions for the phase diagram of $H_{\mathrm{BSB}}$. In scenario (a), $H_{\mathrm{BSB}}$ should flow to strong coupling even for arbitrarily small $U$, causing the system to Néel order for all $U$. In scenario (b), Néel order turns on at a finite order-one coupling through a relativistic quantum critical point. We now provide evidence using unbiased QMC simulations (the method used is reviewed in Ref. [19]) on $H_{\mathrm{BSB}}$ that, as argued above, RG scenario (b) is realized.

For $U / t \gg 1$ we obtain the $S=1 / 2$ Heisenberg model on the BSB, which has Néel order [12]. We focus on the fate of the Néel order as $U$ is weakened. In Fig. 2, we present data for the spin structure factor $S_{\mathrm{AFM}}(\mathbf{k}) \equiv$ $\sum_{\mathbf{r}} e^{i \mathbf{k} \cdot \mathbf{r}}\langle\mathcal{S}(\mathbf{r}) \cdot \mathcal{S}(\mathbf{0})\rangle$, where $\mathcal{S}(\mathbf{r})=\sum_{\ell}\left[\mathbf{S}_{\ell A}(\mathbf{r})-\mathbf{S}_{\ell B}(\mathbf{r})\right]$ is the unit cell antiferromagnetic order parameter, for 


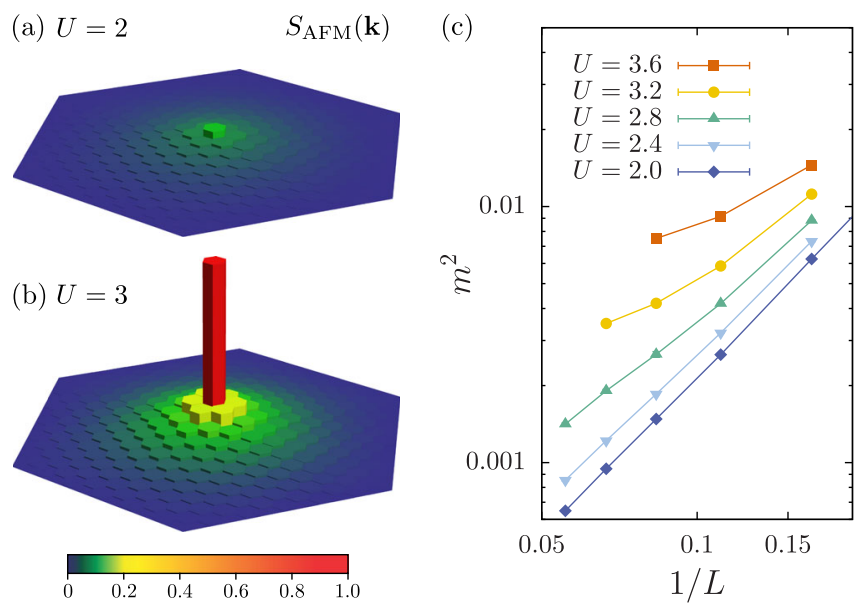

FIG. 2. Structure factor $S_{\mathrm{AFM}}(\mathbf{k})$ and order parameter $m^{2}$ data for $H_{\mathrm{BSB}}$ with $t=t_{\perp}=1$ (fixed throughout the Letter). In (a) and (b), we show the structure factor in $\mathbf{k}$ space for an $L=18$ lattice and its peak at $\boldsymbol{\Gamma}$. Note the dramatic appearance of a Bragg peak structure as one changes from $U=2$ to $U=3$, indicative of a phase transition. (c) Finite size scaling of the order parameter $m^{2} \equiv S_{\mathrm{AFM}}(\boldsymbol{\Gamma}) / L^{2}$, showing a finite $m^{2}$ for $U=3.6$ and 3.2 and then a dramatic suppression for smaller $U$.

various values of $U$. We expect a Néel-ordered state to have a Bragg peak in the spin structure factor at the $\Gamma$ point. As shown in Fig. 2(b) for $U=3$, this is indeed the case. We find in Fig. 2(c) that $m^{2} \equiv S_{\mathrm{AFM}}(\boldsymbol{\Gamma}) / L^{2}$ versus $1 / L$ extrapolates to a finite value, indicating the divergence of the Bragg peak height as expected. As shown in Fig. 2(b), at $U=2$ the peak gets rounded out. Consistent with this rounding out, we find in Fig. 2(c) that $\mathrm{m}^{2}$ appears to extrapolate to zero in the thermodynamic limit for small $U$, indicative of a phase transition.

To study the long-distance ordering quantitatively rather than extrapolate $m^{2}$ (which is notoriously hard when $m^{2}$ is small [20]), we study the correlation ratio $R_{m^{2}}$ of the structure factor at the ordering momentum (the Bragg peak) and the momentum closest to it, $\mathbf{b} / L$ (b is the shortest reciprocal lattice vector):

$$
R_{m^{2}}=1-\frac{S_{\mathrm{AFM}}(\boldsymbol{\Gamma}+\mathbf{b} / L)}{S_{\mathrm{AFM}}(\boldsymbol{\Gamma})} .
$$

This quantity scales to 1 in an ordered phase, scales to 0 in a phase disordered phase, and crosses at a universal value for different $L$ at a quantum critical point, providing a sensitive numerical test for magnetic ordering [21]. The data in Fig. 3 show a crossing at a value of $U_{c} \approx 2.5$ and do not drift significantly on all but the smallest lattices, providing strong evidence for a finite-coupling phase transition (see [15] for more details).

Field theoretic arguments suggest that the transition between the Dirac phase and the Néel phase should be in the GN universality class. The key hallmarks of this kind

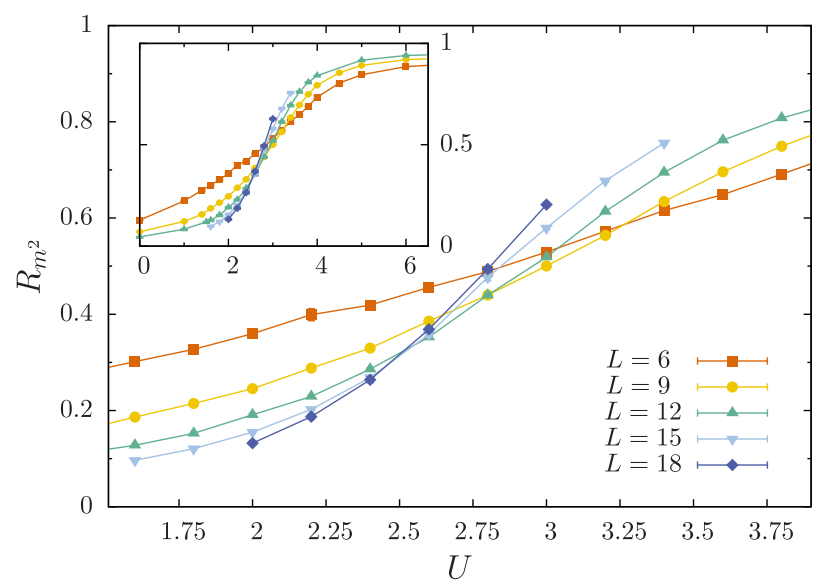

FIG. 3. Correlation ratio $R_{m^{2}}$ [Eq. (3)] close to the phase transition. The inset has a broad range of $U$ showing how $R_{m^{2}}$ reaches its asymptotes of 0 in the nonmagnetic phase and 1 in the magnetic phase. At a continuous transition, $R_{m^{2}}$ is expected to be volume independent and cross at a universal value. The main panel show an enlargement close to the critical point. The crossing point is at $U_{c} \approx 2.5$ with very small drifts on the largest system sizes.

of transition are the gap in the Dirac fermions and the onset of magnetism take place at the same value of $U$ and the emergence of Lorentz invariance or $z=1$ scaling. To test for these features, we study the collapse of the magnetic data $\left(m^{2}\right.$ and $\left.R_{m^{2}}\right)$ and single-particle gap $\Delta_{\text {sp }}$, close to the transition. We extract $\Delta_{\mathrm{sp}}$ from the decay of the imaginary time-displaced Green function using standard methods $[22,23]$. We collapse the magnetic data in Fig. 4, with the scaling forms $m^{2}=L^{a} \mathcal{F}_{m^{2}}\left[L^{1 / \nu}\left(U-U_{c}\right) / U_{c}\right]$ and $R_{m^{2}}=$ $\mathcal{F}_{R_{m^{2}}}\left[L^{1 / \nu}\left(U-U_{c}\right) / U_{c}\right]$, since the $R_{m^{2}}$ has no scaling dimension. Corresponding windows for the parameters that give acceptable collapses are $U_{c}=2.6(1), \nu=0.9(2)$, and

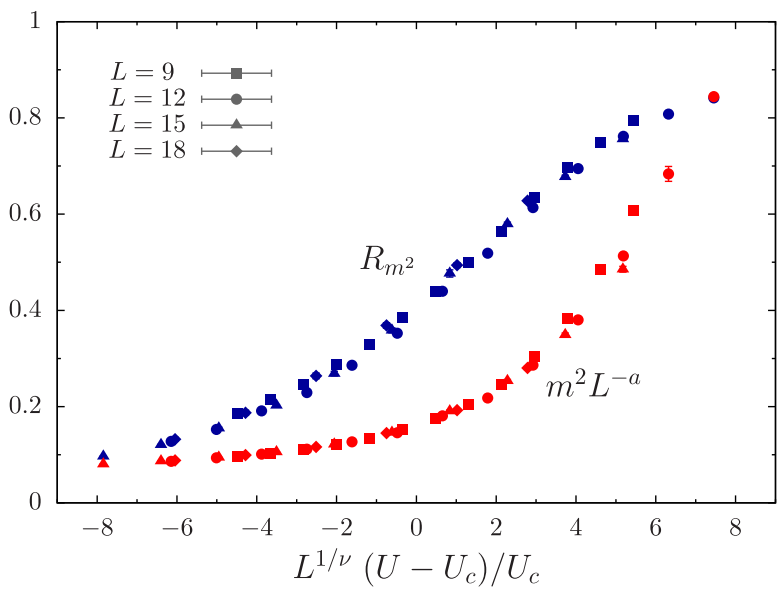

FIG. 4. Collapse of the magnetic data $m^{2}$ and $R_{m^{2}}$ close to the critical point. Parameters used for the collapse are $U_{c}=2.6$, $\nu=0.9$, and $a=0.3$. 


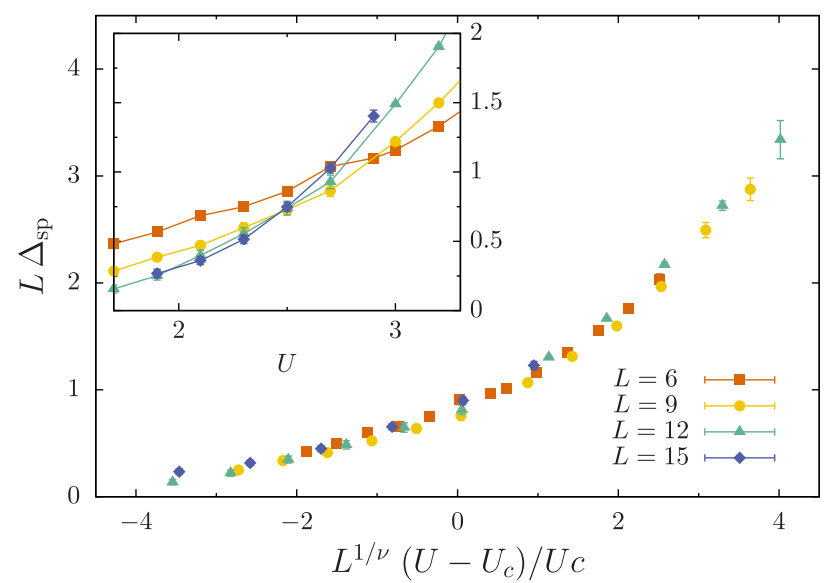

FIG. 5. The collapse of the single-particle gap $L \Delta_{\text {sp }}$ close to the phase transition with the same critical parameters as in Fig. 4 and $z=1$. The inset shows the single-particle gap $L \Delta_{\mathrm{sp}}$ as a function of $U$ which exhibits a crossing at a coupling of $U_{c} \approx 2.5$, consistent with the expected scaling form with $z=1$.

$a=0.3(1)$. For the gap, using a standard finite-size scaling ansatz, we expect $\Delta_{\mathrm{sp}}=L^{-z} \mathcal{F}_{\Delta}\left[L^{1 / \nu}\left(U-U_{c}\right) / U_{c}\right]$. We have verified that our data are consistent with this ansatz with the values of the parameters extracted from the collapse for $R_{m^{2}}$ and $m^{2}$, and $z=1$ in Fig. 5 . Independently, we obtain acceptable collapses for $\Delta_{\mathrm{sp}}$ for the ranges $U_{c}=2.5(2), \nu=1.0(2)$, and $z=0.9(2)$. The inset in Fig. 5 shows our data for $L \Delta_{\text {sp }}$ versus $U$ which should cross at the critical point if $z=1$. We see a crossing in our data which is consistent with the crossings of $R_{m^{2}}$, indicating the existence of a single transition at which both the magnetization turns on and the Dirac fermions get a mass. This is compelling evidence that the transition has $z=1$, in contrast to the $z=2$ scaling at the $Q$ fixed point. Taken together, the identification of the quantum critical point with the GN class provides further evidence that $H_{\mathrm{BSB}}$ realizes the RG flow shown in Fig. 1(b).

We emphasize that our RG flow [Fig. 1(b)] is only schematic: The point marked as $D$ is used to describe collectively fixed points with a linear dispersion but which differ in the number and location of the Dirac cones. Even though we have provided strong evidence for a linear dispersion, we are unable to resolve from our QMC simulations which of these fixed points is realized in $H_{\mathrm{BSB}}$. Our focus here has been on the phase diagram when $\alpha=0$ in the microscopic model; i.e., the free problem has a QBT, such as in $H_{\mathrm{BSB}}$. The evolution of the phase diagram with an explicit trigonal warping term in $H_{\mathrm{BSB}}$ and a more detailed study of the electronic structure will be presented separately.

Our results have important implications for the interpretation of experiments on bilayer graphene. Short-range interactions are the correct model for the experiments in the presence of screening. In this situation, our finding implies that symmetry breaking is not an immediate consequence of interactions, contrary to what has generally been assumed. Indeed, bilayer graphene responds to interactions in much the same way as the single layer: Symmetry breaking will set in only at a finite coupling strength. Making the widely accepted assumptions that interactions are weak and trigonal warping is absent or even weaker, our results predict that bilayer graphene with screened interactions will be in a Dirac phase without any symmetry breaking. We emphasize, however, that while our RG argument applies independently of the form of the short-range interactions, it does not go through in the presence of long-range Coulomb interactions, which by power counting are relevant at the QBT fixed point and hence can plausibly cause a weak coupling instability. Interestingly, this last fact implies that the symmetry breaking that has been detected in the experiment on suspended bilayer graphene is likely a consequence of the long-range tail of the Coulomb interactions [24-26].

We thank F. Assaad, C. L. Kane, C. Honerkamp, A. C. Potter, O. Vafek, S. Wessel, A. Vishwanath, and $\mathrm{K}$. Yang for useful discussions. We acknowledge NSF DMR-1056536 (S.P. and R. K. K.), NSF DMR-1306897 (G. M.), U.S.-Israel BSF 2012120 (G. M.) for financial support, and NSF XSEDE DMR-150037, SuperMUC at Leibniz Supercomputing Centre and JURECA at Jülich Supercomputing Centre (JSC) for generous computer allocations.

[1] A. H. Castro Neto, F. Guinea, N. M. R. Peres, K. S. Novoselov, and A. K. Geim, Rev. Mod. Phys. 81, 109 (2009).

[2] I. F. Herbut, Phys. Rev. Lett. 97, 146401 (2006).

[3] Z. Y. Meng, T. C. Lang, S. Wessel, F. F. Assaad, and A. Muramatsu, Nature (London) 464, 847 (2010).

[4] S. Sorella, Y. Otsuka, and S. Yunoki, Sci. Rep. 2, 992 (2012).

[5] E. McCann and V. I. Fal'ko, Phys. Rev. Lett. 96, 086805 (2006)

[6] K. Sun, H. Yao, E. Fradkin, and S. A. Kivelson, Phys. Rev. Lett. 103, 046811 (2009).

[7] O. Vafek and K. Yang, Phys. Rev. B 81, 041401 (2010).

[8] O. Vafek, Phys. Rev. B 82, 205106 (2010).

[9] F. Zhang, H. Min, M. Polini, and A. H. MacDonald, Phys. Rev. B 81, 041402 (2010).

[10] R. Nandkishore and L. Levitov, Phys. Rev. Lett. 104, 156803 (2010).

[11] Y. Lemonik, I. L. Aleiner, C. Toke, and V. I. Fal'ko, Phys. Rev. B 82, 201408 (2010).

[12] T. C. Lang, Z. Y. Meng, M. M. Scherer, S. Uebelacker, F. F. Assaad, A. Muramatsu, C. Honerkamp, and S. Wessel, Phys. Rev. Lett. 109, 126402 (2012).

[13] V. Cvetkovic, R. E. Throckmorton, and O. Vafek, Phys. Rev. B 86, 075467 (2012).

[14] Our RG argument assumes the model under study has particle-hole symmetry. This symmetry is present in all bipartite models of graphene systems including the $H_{\mathrm{BSB}}$ studied here [Eq. (2)].

[15] See Supplemental Material at http://link.aps.org/ supplemental/10.1103/PhysRevLett.117.086404 for more 
details on the perturbative generation of the linear kinetic term and associated RG flow, as well as additional simulation data and numerical tests.

[16] J. M. Murray and O. Vafek, Phys. Rev. B 89, 201110 (2014).

[17] E. McCann and M. Koshino, Rep. Prog. Phys. 76, 056503 (2013).

[18] Our finite-size QMC results are in numerical agreement with Ref. [12]; where we disagree is on our interpretation: We have provided a more comprehensive analysis of magnetic ordering and the presence of a finite-coupling phase transition using the quantity $R_{m^{2}}$.

[19] F. F. Assaad and H. G. Evertz, Lect. Notes Phys. 739, 277 (2008).
[20] F. Parisen Toldin, M. Hohenadler, F. F. Assaad, and I. F. Herbut, Phys. Rev. B 91, 165108 (2015).

[21] R. K. Kaul, Phys. Rev. Lett. 115, 157202 (2015).

[22] M. Feldbacher and F. F. Assaad, Phys. Rev. B 63, 073105 (2001).

[23] T. C. Lang, Z. Y. Meng, A. Muramatsu, S. Wessel, and F. F. Assaad, Phys. Rev. Lett. 111, 066401 (2013).

[24] B. E. Feldman, J. Martin, and A. Yacoby, Nat. Phys. 5, 889 (2009).

[25] J. Martin, B. E. Feldman, R. T. Weitz, M. T. Allen, and A. Yacoby, Phys. Rev. Lett. 105, 256806 (2010).

[26] R. T. Weitz, M. T. Allen, B. E. Feldman, J. Martin, and A. Yacoby, Science 330, 812 (2010). 